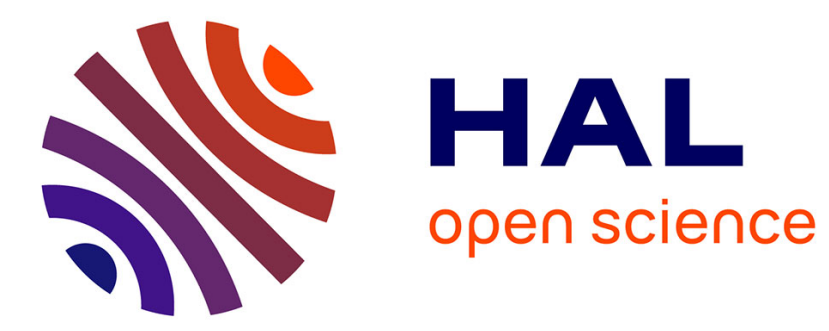

\title{
Search for anomalous couplings in the Higgs sector at LEP
}

\author{
M. Acciarri, P. Achard, O. Adriani, M. Aguilar-Benitez, J. Alcaraz, G.
} Alemanni, J. Allaby, A. Aloisio, M.G. Alviggi, G. Ambrosi, et al.

\section{- To cite this version:}

M. Acciarri, P. Achard, O. Adriani, M. Aguilar-Benitez, J. Alcaraz, et al.. Search for anomalous couplings in the Higgs sector at LEP. Physics Letters B, 2000, 489, pp.102-114. 10.1016/S03702693(00)00890-X . in2p3-00005897

\section{HAL Id: in2p3-00005897 https://hal.in2p3.fr/in2p3-00005897}

Submitted on 2 Oct 2000

HAL is a multi-disciplinary open access archive for the deposit and dissemination of scientific research documents, whether they are published or not. The documents may come from teaching and research institutions in France or abroad, or from public or private research centers.
L'archive ouverte pluridisciplinaire HAL, est destinée au dépôt et à la diffusion de documents scientifiques de niveau recherche, publiés ou non, émanant des établissements d'enseignement et de recherche français ou étrangers, des laboratoires publics ou privés. 
EUROPEAN ORGANIZATION FOR NUCLEAR RESEARCH

CERN-EP/2000-098

July 19, 2000

\title{
Search for anomalous couplings in the Higgs sector at LEP
}

\author{
The L3 Collaboration
}

\begin{abstract}
We search for a Higgs particle with anomalous couplings in the $\mathrm{e}^{+} \mathrm{e}^{-} \rightarrow \mathrm{H} \gamma$, $\mathrm{e}^{+} \mathrm{e}^{-} \rightarrow \mathrm{HZ}$ and $\mathrm{e}^{+} \mathrm{e}^{-} \rightarrow \mathrm{He}^{+} \mathrm{e}^{-}$processes with the L3 detector at LEP. We explore the mass range $70 \mathrm{GeV}<m_{\mathrm{H}}<170 \mathrm{GeV}$ using $176 \mathrm{pb}^{-1}$ of integrated luminosity at a center-of-mass energy of $\sqrt{s}=189 \mathrm{GeV}$. The Higgs decays $\mathrm{H} \rightarrow \mathrm{b} \bar{b}, \mathrm{H} \rightarrow \gamma \gamma$ and $\mathrm{H} \rightarrow \mathrm{Z} \gamma$ are considered in the analysis. No evidence for anomalous Higgs production is found. This is interpreted in terms of limits on the anomalous couplings $d, d_{B}$, $\Delta g_{1}^{\mathrm{Z}}$ and $\Delta \kappa_{\gamma}$. Limits on the $\Gamma(\mathrm{H} \rightarrow \gamma \gamma)$ and $\Gamma(\mathrm{H} \rightarrow \mathrm{Z} \gamma)$ partial widths in the explored Higgs mass range are also obtained.
\end{abstract}

Submitted to Phys. Lett. B 


\section{Introduction}

The spontaneous symmetry breaking mechanism is a fundamental constituent of the Standard Model (SM) [1] of electroweak interactions. Despite its relevance, the experimental information on the Higgs sector of the SM is scarce and indirect at present. The search for the Higgs particle is a key issue for present and future high-energy colliders, and any deviation from expectations could be a clear guide for new physics scenarios beyond the SM.

The SM can be extended via a linear representation of the $S U(2)_{L} \times U(1)_{Y}$ symmetry breaking mechanism [2]. The lowest order representation corresponds to the Standard Model, while at higher orders new interactions between the Higgs particle and gauge bosons become possible. They modify the production mechanisms and decay properties of the Higgs. The relevant CP-invariant Lagrangian terms for neutral bosons are the following [3]:

$$
\begin{aligned}
\mathcal{L}_{\text {eff }}= & g_{\mathrm{H} \gamma \gamma} \mathrm{HA}_{\mu \nu} \mathrm{A}^{\mu \nu}+g_{\mathrm{HZ} \gamma}^{(1)} \mathrm{A}_{\mu \nu} \mathrm{Z}^{\mu} \partial^{\nu} \mathrm{H}+g_{\mathrm{HZ} \gamma}^{(2)} \mathrm{HA}_{\mu \nu} \mathrm{Z}^{\mu \nu} \\
& +g_{\mathrm{HZZ}}^{(1)} \mathrm{Z}_{\mu \nu} \mathrm{Z}^{\mu} \partial^{\nu} \mathrm{H}+g_{\mathrm{HZZ}}^{(2)} \mathrm{HZ}_{\mu \nu} \mathrm{Z}^{\mu \nu}+g_{\mathrm{HZZ}}^{(3)} \mathrm{HZ}_{\mu} \mathrm{Z}^{\mu}
\end{aligned}
$$

where $\mathrm{A}_{\mu}, \mathrm{Z}_{\mu}$ and $\mathrm{H}$ are the photon, $\mathrm{Z}$ and Higgs fields, respectively, and $\mathrm{X}_{\mu \nu}=\partial_{\mu} \mathrm{X}_{\nu}-\partial_{\nu} \mathrm{X}_{\mu}$. The couplings $g_{\mathrm{H} \gamma \gamma}, g_{\mathrm{HZ} \gamma}^{(1)}, g_{\mathrm{HZ} \gamma}^{(2)}, g_{\mathrm{HZZ}}^{(1)}, g_{\mathrm{HZZ}}^{(2)}$ and $g_{\mathrm{HZZ}}^{(3)}$ can be parametrized as follows [4-6]:

$$
\begin{aligned}
g_{\mathrm{H} \gamma \gamma} & =\frac{g}{2 m_{\mathrm{W}}}\left(d \sin ^{2} \theta_{\mathrm{W}}+d_{B} \cos ^{2} \theta_{\mathrm{W}}\right) \\
g_{\mathrm{HZ} \gamma}^{(1)} & =\frac{g}{m_{\mathrm{W}}}\left(\Delta g_{1}^{\mathrm{Z}} \sin 2 \theta_{\mathrm{W}}-\Delta \kappa_{\gamma} \tan \theta_{\mathrm{W}}\right) \\
g_{\mathrm{HZ} \gamma}^{(2)} & =\frac{g}{2 m_{\mathrm{W}}} \sin 2 \theta_{\mathrm{W}}\left(d-d_{B}\right) \\
g_{\mathrm{HZZ}}^{(1)} & =\frac{g}{m_{\mathrm{W}}}\left(\Delta g_{1}^{\mathrm{Z}} \cos 2 \theta_{\mathrm{W}}+\Delta \kappa_{\gamma} \tan ^{2} \theta_{\mathrm{W}}\right) \\
g_{\mathrm{HZZ}}^{(2)} & =\frac{g}{2 m_{\mathrm{W}}}\left(d \cos ^{2} \theta_{\mathrm{W}}+d_{B} \sin ^{2} \theta_{\mathrm{W}}\right) \\
g_{\mathrm{HZZ}}^{(3)} & =\frac{g m_{\mathrm{W}}}{2} \delta_{\mathrm{Z}}
\end{aligned}
$$

where $g$ is the $S U(2)_{L}$ coupling constant, $\theta_{\mathrm{W}}$ is the weak mixing angle and $m_{\mathrm{W}}$ is the W mass. The five anomalous couplings $d, d_{B}, \Delta g_{1}^{\mathrm{Z}}, \Delta \kappa_{\gamma}$ and $\delta_{\mathrm{Z}}$ constitute a convenient set of adimensional parameters to describe deviations in Higgs-vector boson interactions. They are not severely constrained by electroweak measurements at the $\mathrm{Z}$ pole or low energies [3,7]. The couplings $\Delta g_{1}^{\mathrm{Z}}$ and $\Delta \kappa_{\gamma}$ are commonly used in the context of $\mathrm{e}^{+} \mathrm{e}^{-} \rightarrow \mathrm{W}^{+} \mathrm{W}^{-}$studies [5], whereas the couplings $d$ and $d_{B}$ are introduced according to the convention of Reference 4 . Limits on the parameter $\xi=\left(1+\delta_{\mathrm{Z}}\right)$, which quantifies deviations in the magnitude of the HZZ and HWW couplings [6] have already been set [8] and will not be discussed in this paper.

A typical signature of anomalous couplings would be a large cross section for a non-standard Higgs production mechanism such as $\mathrm{e}^{+} \mathrm{e}^{-} \rightarrow \mathrm{H} \gamma$. Another possible effect is the observation of large $\mathrm{H} \rightarrow \gamma \gamma$ and $\mathrm{H} \rightarrow \mathrm{Z} \gamma$ branching fractions, which are zero in the SM at tree level. In addition, a search for anomalous Higgs production with non-zero $\Delta g_{1}^{Z}$ or $\Delta \kappa_{\gamma}$ couplings offers a complementary way to look for the same type of deviations which may be present in the $\mathrm{e}^{+} \mathrm{e}^{-} \rightarrow \mathrm{W}^{+} \mathrm{W}^{-}$process.

The data used in this analysis were collected with the L3 detector [9] at a center-of-mass energy of $\sqrt{s}=189 \mathrm{GeV}$ and correspond to an integrated luminosity of $176 \mathrm{pb}^{-1}$. Previous experimental analyses on anomalous Higgs production accompanied by photons are discussed in Reference 10. 


\section{Analysis strategy and Monte Carlo samples}

We search for a Higgs particle emitted in the $\mathrm{e}^{+} \mathrm{e}^{-} \rightarrow \mathrm{H} \gamma$ and $\mathrm{e}^{+} \mathrm{e}^{-} \rightarrow \mathrm{He}^{+} \mathrm{e}^{-}$processes. These processes may be enhanced by the presence of anomalous $\mathrm{H} \gamma \gamma$ and $\mathrm{HZ} \gamma$ couplings and have sensitivity to Higgs masses up to the center-of-mass energy of the collision $\left(m_{\mathrm{H}}<\sqrt{s}\right)$. The analysis is complemented with a study of the $\mathrm{e}^{+} \mathrm{e}^{-} \rightarrow \mathrm{HZ}$ process, which is sensitive to anomalous HZZ and HZ $\gamma$ couplings for Higgs masses below the kinematic limit: $m_{\mathrm{H}}<\sqrt{s}-m_{\mathrm{Z}}$. The Feynman diagrams for these three anomalous processes are shown in Figure 1.

In order to search for the anomalous $\mathrm{e}^{+} \mathrm{e}^{-} \rightarrow \mathrm{H} \gamma$ process (Figure 1a) a dedicated generator is written. It implements the expected $\left(1+\cos ^{2} \theta_{\mathrm{H}}\right) d\left(\cos \theta_{\mathrm{H}}\right)$ dependence for the differential cross section as a function of the Higgs production angle, $\theta_{\mathrm{H}}$. The generator takes into account initial state radiation [11], photon emission by final-state particles [12], spin correlations and off-shell effects in cascade decays like $\mathrm{H} \rightarrow \mathrm{Z} \gamma \rightarrow \mathrm{f} \bar{f} \gamma$.

The $\mathrm{e}^{+} \mathrm{e}^{-} \rightarrow \mathrm{He}^{+} \mathrm{e}^{-}$process (Figure $1 \mathrm{~b}$ ) is interpreted as the production of a narrow-width spin-zero resonance (the Higgs particle) in two-photon processes. For the generation of this process, the PC generator [13] is used. The $\mathrm{e}^{+} \mathrm{e}^{-} \rightarrow$ HZ process, which is also affected by the presence of anomalous couplings [14] (Figure 1c), is studied by reinterpreting the cross section limits obtained from the L3 SM [15] and fermiophobic [16] Higgs searches.

The branching fractions and partial widths of an anomalous Higgs are determined according to the calculations of References 17 and 18. The search is restricted to the Higgs mass range $70 \mathrm{GeV}<m_{\mathrm{H}}<170 \mathrm{GeV}$. The decay channels $\mathrm{H} \rightarrow \gamma \gamma, \mathrm{H} \rightarrow \mathrm{Z} \gamma$ and $\mathrm{H} \rightarrow \mathrm{b} \overline{\mathrm{b}}$ are considered. The cases $\mathrm{H} \rightarrow \gamma \gamma$ and $\mathrm{H} \rightarrow \mathrm{Z} \gamma$ complement each other in sensitivity, probing a large part of the parameter space. The $\mathrm{H} \rightarrow \mathrm{b} \bar{b}$ decay is dominant in the parameter region where $\mathrm{H} \rightarrow \gamma \gamma$ is strongly suppressed and $\mathrm{H} \rightarrow \mathrm{Z} \gamma$ is kinematically forbidden.

The analysis is performed as a function of the Higgs mass hypothesis. Signal events are generated for the following six Higgs masses: 70, 90, 110, 130, 150 and $170 \mathrm{GeV}$. For each mass under consideration and for each possible production and decay mode, more than 2000 signal events are generated. For the study of SM backgrounds the following generators are considered: KK2F [19] and PYTHIA [20] for $\mathrm{e}^{+} \mathrm{e}^{-} \rightarrow \mathrm{q} \overline{\mathrm{q}}(\gamma)$ contaminations, PYTHIA for multiperipheral $\mathrm{e}^{+} \mathrm{e}^{-} \rightarrow \mathrm{e}^{+} \mathrm{e}^{-} \mathrm{q} \overline{\mathrm{q}}$ events, GGG [21] for $\mathrm{e}^{+} \mathrm{e}^{-} \rightarrow \gamma \gamma(\gamma)$, KORALW [22] for $\mathrm{e}^{+} \mathrm{e}^{-} \rightarrow$ $\mathrm{W}^{+} \mathrm{W}^{-}$, EXCALIBUR [23] for $\mathrm{e}^{+} \mathrm{e}^{-} \rightarrow \mathrm{We} \nu$ and remaining four-fermion backgrounds at high invariant masses, and KORALZ [24] for $\mathrm{e}^{+} \mathrm{e}^{-} \rightarrow \tau^{+} \tau^{-}(\gamma)$. In all cases the Monte Carlo (MC) statistics used in the analysis is more than 10 times the statistics present in data, except for multiperipheral $\mathrm{e}^{+} \mathrm{e}^{-} \rightarrow \mathrm{e}^{+} \mathrm{e}^{-} \mathrm{q} \overline{\mathrm{q}}$ events, for which the relative factor is two.

All MC samples are simulated in the L3 detector and reconstructed in the same way as data. Time-dependent detector inefficiencies are taken into account.

\section{Event selection}

The $\mathrm{e}^{+} \mathrm{e}^{-} \rightarrow \mathrm{H} \gamma$ and $\mathrm{e}^{+} \mathrm{e}^{-} \rightarrow \mathrm{He}^{+} \mathrm{e}^{-}$Higgs production mechanisms lead to characteristic topologies. In the $\mathrm{e}^{+} \mathrm{e}^{-} \rightarrow \mathrm{H} \gamma$ process, a high-energy photon of fixed energy is produced, and typically most of the collision energy is visible in the final state. Events originating from a twophoton collision, $\mathrm{e}^{+} \mathrm{e}^{-} \rightarrow \mathrm{He}^{+} \mathrm{e}^{-}$, have missing longitudinal momentum and missing mass, as the two emerging electrons tend to escape detection at very low polar angles. The analysis of the specific channel $\mathrm{e}^{+} \mathrm{e}^{-} \rightarrow \mathrm{H} \gamma, \mathrm{H} \rightarrow \mathrm{Z} \gamma$, is based on the $\mathrm{e}^{+} \mathrm{e}^{-} \rightarrow \mathrm{Z} \gamma \gamma$ selection criteria described in Reference 25. All analyses are performed in Higgs mass steps of $1 \mathrm{GeV}$ by interpolation of the generated Higgs signal efficiencies. This is particularly relevant in the case of photonic 
Higgs decays, where the good energy resolution of the L3 detector can be exploited efficiently.

Most of the analyses rely on photon identification. A photon is defined as a shower in the electromagnetic calorimeter with a profile consistent with that of an electromagnetic particle and no associated track in the vertex chamber. The photon candidates must satisfy $E_{\gamma}>5 \mathrm{GeV}$ and $\left|\cos \theta_{\gamma}\right|<0.8$, where $E_{\gamma}$ is the photon energy and $\theta_{\gamma}$ is its polar angle. These cuts reduce the background associated to initial and final-state radiation, while keeping a large efficiency for the Higgs signal. The identification of $\mathrm{H} \rightarrow \mathrm{b} \bar{b}$ decays is also crucial in this study. The b-tagging performance is similar to the one obtained in the L3 SM Higgs search [26].

\section{$3.1 \quad \mathrm{e}^{+} \mathrm{e}^{-} \rightarrow \mathrm{H} \gamma \rightarrow \gamma \gamma \gamma \quad$ analysis}

In order to select $\mathrm{e}^{+} \mathrm{e}^{-} \rightarrow \mathrm{H} \gamma \rightarrow \gamma \gamma \gamma$ events we require three photon candidates with a total electromagnetic energy larger than $\sqrt{s} / 2$. In addition, the invariant mass of at least one of the photon pairs in the event must be consistent with the Higgs mass hypothesis within $3 \mathrm{GeV}$.

After these cuts the contamination from processes other than $\mathrm{e}^{+} \mathrm{e}^{-} \rightarrow \gamma \gamma(\gamma)$ is estimated to be negligible. Figure 2a presents the distribution of the invariant mass in data and in $\mathrm{MC}$ for one of the Higgs mass hypotheses with largest signal significance.

The number of expected and observed events, the signal efficiency in the full phase space, and the limit on $\sigma\left(\mathrm{e}^{+} \mathrm{e}^{-} \rightarrow \mathrm{H} \gamma\right) \operatorname{Br}(\mathrm{H} \rightarrow \gamma \gamma)$ are shown in Table 1 for several Higgs mass hypotheses. No evidence for any anomalous signal is observed, leading to the $95 \%$ confidence level (CL) limits shown in Figure 3. Throughout this paper, a Bayesian approach with a flat prior distribution is adopted in the derivation of the limits on the signal cross section.

\begin{tabular}{|c|c|c|c|c|}
\hline $\begin{array}{c}\mathrm{m}_{\mathrm{H}} \\
(\mathrm{GeV})\end{array}$ & $\begin{array}{c}\text { Data } \\
\text { events }\end{array}$ & $\begin{array}{c}\text { Background } \\
\text { events }\end{array}$ & $\begin{array}{c}\text { Signal } \\
\text { acceptance }(\%)\end{array}$ & $\begin{array}{c}\text { 95\% CL Upper Limit on } \\
\sigma\left(\mathrm{e}^{+} \mathrm{e}^{-} \rightarrow \mathrm{H} \gamma\right) \mathrm{Br}(\mathrm{H} \rightarrow \gamma \gamma)(\mathrm{fb})\end{array}$ \\
\hline 70 & 0 & 1.3 & 22.2 & 70 \\
90 & 1 & 0.8 & 20.8 & 115 \\
110 & 1 & 0.8 & 19.7 & 122 \\
130 & 0 & 0.8 & 18.9 & 91 \\
150 & 0 & 0.9 & 18.5 & 152 \\
170 & 2 & 1.9 & 18.4 & 93 \\
\hline
\end{tabular}

Table 1: Number of selected candidates, background events, signal acceptance and 95\% CL cross section limits after the $\mathrm{e}^{+} \mathrm{e}^{-} \rightarrow \mathrm{H} \gamma \rightarrow \gamma \gamma \gamma$ selection for different Higgs mass hypotheses.

\section{$3.2 \quad \mathrm{e}^{+} \mathrm{e}^{-} \rightarrow \mathrm{H} \gamma \rightarrow \mathrm{b} \overline{\mathrm{b}} \gamma \quad$ analysis}

High particle multiplicity and momentum imbalance cuts are applied in order to select an initial sample of hadronic events. A b $\bar{b} \gamma$ event is tagged by the presence of an isolated photon and b-hadrons (the event b-tag discriminant, $B_{\text {tag }}[26]$, must exceed 1.5).

The contamination in the selected sample is dominated by the almost irreducible $\mathrm{e}^{+} \mathrm{e}^{-} \rightarrow \mathrm{Z} \gamma$ process. The remaining backgrounds are estimated to be at the $1 \%$ level. The number of expected and observed events and the signal acceptance in the full phase space are shown in Table 2 for several Higgs mass hypotheses.

The distribution of the mass recoiling to the photon in data and in $\mathrm{MC}$ for one of the Higgs mass hypotheses with largest signal significance is plotted in Figure 2b. This distribution is 
used to set upper limits on the magnitude of $\sigma\left(\mathrm{e}^{+} \mathrm{e}^{-} \rightarrow \mathrm{H} \gamma\right) \operatorname{Br}(\mathrm{H} \rightarrow \mathrm{b} \overline{\mathrm{b}})$. There is good agreement between data and MC in the absence of a Higgs signal, leading to the limits shown in Table 2 and Figure 3.

\begin{tabular}{|c|c|c|c|c|}
\hline $\begin{array}{c}\mathrm{m}_{\mathrm{H}} \\
(\mathrm{GeV})\end{array}$ & $\begin{array}{c}\text { Data } \\
\text { events }\end{array}$ & $\begin{array}{c}\text { Background } \\
\text { events }\end{array}$ & $\begin{array}{c}\text { Signal } \\
\text { acceptance }(\%)\end{array}$ & $\begin{array}{c}\text { 95\% CL Upper Limit on } \\
\sigma\left(\mathrm{e}^{+} \mathrm{e}^{-} \rightarrow \mathrm{H} \gamma\right) \mathrm{Br}(\mathrm{H} \rightarrow \mathrm{bb})(\mathrm{fb})\end{array}$ \\
\hline 70 & 2 & 2.8 & 20.8 & 101 \\
90 & 77 & 83.7 & 24.7 & 365 \\
110 & 21 & 20.7 & 22.5 & 200 \\
130 & 9 & 10.7 & 20.2 & 155 \\
150 & 3 & 10.3 & 18.8 & 105 \\
170 & 10 & 16.0 & 17.6 & 157 \\
\hline
\end{tabular}

Table 2: Number of selected candidates, background events, signal acceptances and cross section limits after the $\mathrm{e}^{+} \mathrm{e}^{-} \rightarrow \mathrm{H} \gamma \rightarrow \mathrm{b} \bar{b} \gamma$ selection for different Higgs mass hypotheses.

\section{3 $\quad \mathrm{e}^{+} \mathrm{e}^{-} \rightarrow \mathrm{H} \gamma \rightarrow \mathrm{Z} \gamma \gamma \quad$ analysis}

The selection criteria for this channel are the same as those used for the measurement of the $\mathrm{e}^{+} \mathrm{e}^{-} \rightarrow \mathrm{H} \gamma \rightarrow \mathrm{Z} \gamma \gamma$ cross section [25]. We select 36 events, in agreement with the expected SM background of 39.2. The signal efficiency over the full phase space and the upper limits on $\sigma\left(\mathrm{e}^{+} \mathrm{e}^{-} \rightarrow \mathrm{H} \gamma\right) \operatorname{Br}(\mathrm{H} \rightarrow \mathrm{Z} \gamma)$ are shown in Table 3 and Figure 3 .

\begin{tabular}{|c|c|c|}
\hline $\begin{array}{c}\mathrm{m}_{\mathrm{H}} \\
(\mathrm{GeV})\end{array}$ & $\begin{array}{c}\text { Signal } \\
\text { acceptance }(\%)\end{array}$ & $\begin{array}{c}95 \% \mathrm{CL} \text { Upper Limit on } \\
\sigma\left(\mathrm{e}^{+} \mathrm{e}^{-} \rightarrow \mathrm{H} \gamma\right) \mathrm{Br}(\mathrm{H} \rightarrow \mathrm{Z} \gamma)(\mathrm{fb})\end{array}$ \\
\hline 95 & 9.3 & 723 \\
100 & 28.6 & 234 \\
110 & 33.5 & 200 \\
130 & 36.0 & 186 \\
150 & 34.5 & 194 \\
170 & 34.2 & 196 \\
\hline
\end{tabular}

Table 3: Signal acceptances and cross section limits after the $\mathrm{e}^{+} \mathrm{e}^{-} \rightarrow \mathrm{H} \gamma \rightarrow \mathrm{Z} \gamma \gamma$ selection for different Higgs mass hypotheses.

\section{$3.4 \quad \mathrm{e}^{+} \mathrm{e}^{-} \rightarrow \mathrm{He}^{+} \mathrm{e}^{-}, \mathrm{H} \rightarrow \gamma \gamma$ analysis}

The selection of the $\mathrm{e}^{+} \mathrm{e}^{-} \rightarrow \mathrm{e}^{+} \mathrm{e}^{-} \mathrm{H} \rightarrow \mathrm{e}^{+} \mathrm{e}^{-} \gamma \gamma$ signal requires the presence of two photon candidates in the event. In addition, a kinematic fit to the signal hypothesis is performed. We assume that all the missing energy is lost in the beam pipe and that the visible mass of the event is consistent with $m_{\mathrm{H}}$ within the experimental uncertainties. Finally, a cut on the $\chi^{2}$ of this fit is applied.

The distribution of the two photon invariant mass for data and MC after the kinematic fit is shown in Figure 2c for one of the Higgs mass hypotheses. The background is dominated by $\mathrm{e}^{+} \mathrm{e}^{-} \rightarrow \gamma \gamma(\gamma)$ events. Since $\sigma\left(\mathrm{e}^{+} \mathrm{e}^{-} \rightarrow \mathrm{He}^{+} \mathrm{e}^{-}\right)$is proportional to the partial Higgs width 
into photons, $\Gamma(\mathrm{H} \rightarrow \gamma \gamma)$, the cross section limits are directly interpreted in terms of $\Gamma(\mathrm{H} \rightarrow$ $\gamma \gamma) \operatorname{Br}(\mathrm{H} \rightarrow \gamma \gamma)$. The number of expected and observed events and the signal efficiency over the full phase space are listed in Table 4 for several Higgs mass hypotheses. There is no evidence for any anomalous signal, leading to the 95\% CL limits presented in Table 4 and in Figure 4.

\begin{tabular}{|c|c|c|c|c|}
\hline $\begin{array}{c}\mathrm{m}_{\mathrm{H}} \\
(\mathrm{GeV})\end{array}$ & $\begin{array}{c}\text { Data } \\
\text { events }\end{array}$ & $\begin{array}{c}\text { Background } \\
\text { events }\end{array}$ & $\begin{array}{c}\text { Signal } \\
\text { acceptance }(\%)\end{array}$ & $\begin{array}{c}\text { 95\% CL Upper Limit on } \\
\Gamma(\mathrm{H} \rightarrow \gamma \gamma) \mathrm{Br}(\mathrm{H} \rightarrow \gamma \gamma)(\mathrm{MeV})\end{array}$ \\
\hline 70 & 0 & 0.0 & 22.9 & 0.3 \\
90 & 0 & 1.1 & 27.5 & 0.8 \\
110 & 4 & 2.6 & 31.4 & 4.5 \\
130 & 6 & 4.8 & 34.7 & 11.6 \\
150 & 10 & 9.9 & 37.3 & 32.7 \\
170 & 19 & 28.7 & 39.2 & 88.7 \\
\hline
\end{tabular}

Table 4: Number of selected candidates, background events, signal acceptances and limits on $\Gamma(\mathrm{H} \rightarrow \gamma \gamma) \operatorname{Br}(\mathrm{H} \rightarrow \gamma \gamma)$ from the $\mathrm{e}^{+} \mathrm{e}^{-} \rightarrow \mathrm{e}^{+} \mathrm{e}^{-} \mathrm{H} \rightarrow \mathrm{e}^{+} \mathrm{e}^{-} \gamma \gamma$ selection for different Higgs mass hypotheses.

\section{$3.5 \mathrm{e}^{+} \mathrm{e}^{-} \rightarrow \mathrm{He}^{+} \mathrm{e}^{-}, \mathrm{H} \rightarrow \mathrm{b} \overline{\mathrm{b}}$ analysis}

Particle multiplicity and transverse momentum imbalance cuts are applied in order to select an initial sample of hadronic events. Most of the $\mathrm{e}^{+} \mathrm{e}^{-} \rightarrow \mathrm{e}^{+} \mathrm{e}^{-} \mathrm{q} \overline{\mathrm{q}}$ background is rejected by requiring a visible event mass greater than $50 \mathrm{GeV}$. The b-quark purity is increased by a cut on the the event tag discriminant $\left(B_{\operatorname{tag}}>2\right)$. Events are constrained to two jets by means of the Durham algorithm [27]. Those events with a $y_{23}$ value in excess of 0.05 are rejected, where $y_{23}$ is the jet resolution parameter for which the transition from two to three jets occurs. As for the $\mathrm{e}^{+} \mathrm{e}^{-} \rightarrow \mathrm{e}^{+} \mathrm{e}^{-} \mathrm{H} \rightarrow \mathrm{e}^{+} \mathrm{e}^{-} \gamma \gamma$ case, a kinematic fit is performed. A final cut on missing mass after the kinematic fit, $M_{m i s s}$, is required in order to reject a large fraction of the $\mathrm{e}^{+} \mathrm{e}^{-} \rightarrow \mathrm{b} \overline{\mathrm{b}} \gamma$ background. For Higgs masses above $95 \mathrm{GeV}$, we require $M_{\text {miss }}>0.09 \sqrt{s}$. For lower Higgs masses this requirement is tightened to $M_{\text {miss }}>0.44 \sqrt{s}$ to further reject the $\mathrm{e}^{+} \mathrm{e}^{-} \rightarrow \mathrm{Z} \gamma$ contamination. Figure $2 \mathrm{~d}$ shows the invariant mass distribution of the selected events for the $m_{\mathrm{H}}=m_{\mathrm{Z}}$ hypothesis after kinematic fit.

After these cuts, the remaining background corresponds mostly to $\mathrm{e}^{+} \mathrm{e}^{-} \rightarrow \mathrm{q} \overline{\mathrm{q}}(\gamma)$ events, with a small contribution from $\mathrm{e}^{+} \mathrm{e}^{-} \rightarrow \mathrm{W}^{+} \mathrm{W}^{-}$and $\mathrm{e}^{+} \mathrm{e}^{-} \rightarrow \mathrm{e}^{+} \mathrm{e}^{-} \mathrm{q} \overline{\mathrm{q}}$ events. The $\chi^{2}$ distribution of the kinematic fit shows the largest sensitivity to the signal and is used to set the limits on $\Gamma(\mathrm{H} \rightarrow \gamma \gamma) \operatorname{Br}(\mathrm{H} \rightarrow \mathrm{b} \bar{b})$ presented in Table 5 and Figure 4.

\section{Limits on anomalous couplings}

The analyses performed over the different Higgs production mechanisms and decay channels show that the experimental data agree with the SM MC predictions. This agreement is quantified in terms of limits on the anomalous parameters $d, d_{B}, \Delta g_{1}^{Z}$ and $\Delta \kappa_{\gamma}$. 


\begin{tabular}{|c|c|c|c|c|}
\hline $\begin{array}{c}\mathrm{m}_{\mathrm{H}} \\
(\mathrm{GeV})\end{array}$ & $\begin{array}{c}\text { Data } \\
\text { events }\end{array}$ & $\begin{array}{c}\text { Background } \\
\text { events }\end{array}$ & $\begin{array}{c}\text { Signal } \\
\text { acceptance }(\%)\end{array}$ & $\begin{array}{c}\text { 95\% CL Upper Limit on } \\
\Gamma(\mathrm{H} \rightarrow \gamma \gamma) \operatorname{Br}(\mathrm{H} \rightarrow \mathrm{b})(\mathrm{MeV})\end{array}$ \\
\hline 70 & 188 & 182.3 & 15.4 & 2.7 \\
90 & 107 & 112.9 & 16.5 & 6.6 \\
110 & 222 & 232.6 & 28.7 & 19.7 \\
130 & 298 & 323.9 & 28.3 & 43.1 \\
150 & 388 & 399.6 & 29.9 & 90.9 \\
170 & 367 & 369.3 & 28.4 & 462.7 \\
\hline
\end{tabular}

Table 5: Number of selected candidates, background events, signal acceptances and limits on $\Gamma(\mathrm{H} \rightarrow \gamma \gamma) \operatorname{Br}(\mathrm{H} \rightarrow \mathrm{b} \bar{b})$ from the $\mathrm{e}^{+} \mathrm{e}^{-} \rightarrow \mathrm{e}^{+} \mathrm{e}^{-} \mathrm{H} \rightarrow \mathrm{e}^{+} \mathrm{e}^{-} \mathrm{b} \overline{\mathrm{b}}$ selection for different Higgs mass hypotheses.

\subsection{One-dimensional limits}

Exclusion limits for each individual coupling are derived as a function of the Higgs mass following criteria similar to the ones employed in the SM Higgs search [8]. For a given coupling $x$, a point in the $\left(m_{\mathrm{H}}, x\right)$ plane is considered as excluded at the $95 \%$ CL or more if the ratio of the confidence level for the "signal+background" hypothesis to the confidence level for the "background-only" hypothesis is less than 0.05. In this study all other couplings are assumed to be zero.

The results for the four parameters: $d, d_{B}, \Delta g_{1}^{\mathrm{Z}}$ and $\Delta \kappa_{\gamma}$ are shown in Figures 5 and 6 . In addition to the combined results obtained using all processes under study, the individual results for the most sensitive channels are also displayed.

In all cases, the $\mathrm{e}^{+} \mathrm{e}^{-} \rightarrow \mathrm{HZ}$ searches are enough to exclude the region $m_{\mathrm{H}} \lesssim \sqrt{s}-m_{\mathrm{Z}}$ for any value of the anomalous coupling. The fermiophobic $\mathrm{e}^{+} \mathrm{e}^{-} \rightarrow \mathrm{HZ}, \mathrm{H} \rightarrow \gamma \gamma$ search is sensitive to large values of $d$ and $d_{B}$, for which there is an enhancement of the $\mathrm{H} \rightarrow \gamma \gamma$ branching fraction. The standard search for $\mathrm{e}^{+} \mathrm{e}^{-} \rightarrow \mathrm{HZ}, \mathrm{H} \rightarrow \mathrm{b} \bar{b}, \tau^{+} \tau^{-}$covers the region $d \approx d_{B} \approx 0$.

The $\mathrm{e}^{+} \mathrm{e}^{-} \rightarrow \mathrm{H} \gamma \rightarrow \gamma \gamma \gamma$ and $\mathrm{e}^{+} \mathrm{e}^{-} \rightarrow \mathrm{e}^{+} \mathrm{e}^{-} \mathrm{H} \rightarrow \mathrm{e}^{+} \mathrm{e}^{-} \gamma \gamma$ channels are sensitive to large values of $\mathrm{H} \gamma \gamma$ couplings, i.e. to large values of the combination $d \sin ^{2} \theta_{\mathrm{W}}+d_{B} \cos ^{2} \theta_{\mathrm{W}}$ (Figure 5). On the contrary, the $\mathrm{e}^{+} \mathrm{e}^{-} \rightarrow \mathrm{H} \gamma \rightarrow \mathrm{Z} \gamma \gamma$ process has a dominant role when $\mathrm{H} \rightarrow \gamma \gamma$ is suppressed (Figure 6). This is the case for the fits to $\Delta g_{1}^{Z}$ and $\Delta \kappa_{\gamma}$ in the Higgs mass range $m_{\mathrm{Z}}<\sqrt{s}<2 m_{\mathrm{W}}$. The sensitivity of the $\mathrm{e}^{+} \mathrm{e}^{-} \rightarrow \mathrm{H} \gamma \rightarrow \mathrm{b} \overline{\mathrm{b}} \gamma$ channel (Figure 6 at $m_{\mathrm{H}} \approx m_{\mathrm{Z}}$ ) concerns the region in which $\mathrm{H} \rightarrow \gamma \gamma$ is small, the $\mathrm{HZ} \gamma$ coupling is large and the $\mathrm{H} \rightarrow \mathrm{Z} \gamma$ decay is kinematically not possible. The reduced sensitivity of the $\mathrm{e}^{+} \mathrm{e}^{-} \rightarrow \mathrm{e}^{+} \mathrm{e}^{-} \mathrm{H} \rightarrow \mathrm{e}^{+} \mathrm{e}^{-} \mathrm{b} \overline{\mathrm{b}}$ process is due to the strong decrease of the $\mathrm{H} \rightarrow \mathrm{b} \bar{b}$ branching fraction in the presence of large $\mathrm{H} \gamma \gamma$ couplings.

The sensitivity of the analysis degrades rapidly when $m_{\mathrm{H}}$ approaches the $2 m_{\mathrm{W}}$ threshold, where the $\mathrm{H} \rightarrow \mathrm{W}^{+} \mathrm{W}^{-}$decay becomes dominant even in the presence of relatively large anomalous couplings.

Another usual assumption [3] is to consider that all anomalous interactions have the same strength $F$ at the scale of new physics $\Lambda$, i.e. $m_{\mathrm{W}}^{2} F / \Lambda^{2}=\Delta \kappa_{\gamma}=-d=-d_{B} / \tan ^{2} \theta_{\mathrm{W}}=$ $2 \cos ^{2} \theta_{\mathrm{W}} \Delta g_{1}^{\mathrm{Z}}$. This choice, although reasonable in what concerns orders of magnitude, is very particular. It implies the absence of an anomalous $\mathrm{H} \rightarrow \mathrm{Z} \gamma$ decay and a large exclusion power for the channels sensitive to the $\mathrm{H} \gamma \gamma$ coupling. We show the excluded regions under this assumption in Figures 5 and 6. 


\subsection{Two-dimensional limits}

The $95 \%$ CL contours obtained from a likelihood fit in the $\left(d, d_{B}\right)$ plane, taking into account all analyzed processes, are shown in Figure 7 for different Higgs masses. In this fit we assume that the couplings $\Delta g_{1}^{Z}$ and $\Delta \kappa_{\gamma}$ are zero. The $\mathrm{e}^{+} \mathrm{e}^{-} \rightarrow \mathrm{H} \gamma \rightarrow \mathrm{Z} \gamma \gamma$ process helps in excluding large values of the anomalous couplings in the region where $d \sin ^{2} \theta_{\mathrm{W}}+d_{B} \cos ^{2} \theta_{\mathrm{W}} \approx 0$. The fit is reinterpreted as a fit in the $(\Gamma(\mathrm{H} \rightarrow \gamma \gamma), \Gamma(\mathrm{H} \rightarrow \mathrm{Z} \gamma))$ plane. The results are also presented in Figure 7 for different Higgs masses.

\section{Acknowledgements}

We wish to express our gratitude to the CERN accelerator divisions for the excellent performance of the LEP machine. We acknowledge the contributions of the engineers and technicians who have participated in the construction and maintenance of this experiment.

\section{References}

[1] S.L. Glashow, Nucl. Phys. 22 (1961) 579;

S. Weinberg, Phys. Rev. Lett. 19 (1967) 1264;

A. Salam, "Elementary Particle Theory", Ed. N. Svartholm, Stockholm, Almqvist and Wiksell (1968), 367;

P.W. Higgs, Phys. Lett. 12 (1964) 132.

[2] W. Buchmüller and D. Wyler, Nucl. Phys. B 268, (1986) 621;

C.J.C. Burgess and H.J. Schnitzer, Nucl. Phys. B 228 (1983) 424;

C.N. Leung, S.T. Love and S. Rao, Z. Phys. C 31 (1986) 433.

[3] O.J.P. Éboli et al., Phys. Lett. B 434 (1998) 340;

M.C. González-García, Int. J. Mod. Phys. A 14 (1999) 3121.

[4] G.J. Gounaris, F.M. Renard and N.D. Vlachos, Nucl. Phys. B 459 (1996) 51.

[5] K. Hagiwara et al., Nucl. Phys. B 282 (1987) 253.

[6] B. Grzạdkowski and J. Wudka, Phys. Lett. B 364 (1995) 49.

[7] S. Alam, S. Dawson and R. Szalapski, Phys. Rev. D 57 (1998) 1577.

[8] The ALEPH, DELPHI, L3 and OPAL Collaborations and the LEP Working Group for Higgs boson Searches, Preprint CERN-EP-2000-055 (2000).

[9] B.Adeva et al., L3 Collaboration, Nucl. Inst. Meth. A289 (1990) 35;

J.A.Bakken et al., Nucl. Inst. Meth. A275 (1989) 81;

O.Adriani et al., Nucl. Inst. Meth. A302 (1991) 53;

B.Adeva et al., Nucl. Inst. Meth. A323 (1992) 109;

K.Deiters et al., Nucl. Inst. Meth. A323 (1992) 162;

M.Chemarin et al., Nucl. Inst. Meth. A349 (1994) 345;

G.Basti et al., Nucl. Inst. Meth. A374 (1996) 293;

A.Adam et al., Nucl. Inst. Meth. A383 (1996) 342. 
[10] DELPHI Collaboration, P. Abreu et al., Phys. Lett. B 458 (1999) 431.

[11] F.A. Berends and R. Kleiss, Nucl. Phys. B 260 (1985) 32.

[12] E. Barberio and Z. Wạs, Comp. Phys. Comm. 79 (1994) 291.

[13] F.L. Linde, "Charm Production in Two-Photon Collisions", Ph.D. Thesis, Rijksuniversiteit Leiden 1988.

[14] K. Hagiwara and M.L. Stong, Z. Phys. C 62 (1994) 99.

[15] L3 Collaboration, M. Acciarri et al., Phys. Lett. B 461 (1999) 376.

[16] L3 Collaboration, M. Acciarri et al., Preprint CERN-EP-2000-103 (2000).

[17] K. Hagiwara, R. Szalapski and D. Zeppenfeld, Phys. Lett. B 318 (1993) 155.

[18] J.C. Romao and S. Andringa, Eur. Phys. J. C7 (1999) 631.

[19] S. Jadach, B.F.L. Ward and Z. Wạs, Preprint hep-ph/9912214 (1999).

[20] T. Sjöstrand, Comp. Phys. Comm. 82 (1994) 74.

[21] F. A. Berends and R. Kleiss, Nucl. Phys. B 186 (1981) 22.

[22] M. Skrzypek et al., Comp. Phys. Comm. 94 (1996) 216;

M. Skrzypek et al., Phys. Lett. B 372 (1996) 289.

[23] F.A. Berends, R. Kleiss and R. Pittau, Comp. Phys. Comm. 85 (1995) 437.

[24] S. Jadach, B. F. L. Ward and Z. Wạs, Comp. Phys. Comm. 79 (1994) 503.

[25] L3 Collaboration, M. Acciarri et al., Phys. Lett. B 478 (2000) 39.

[26] L3 Collaboration, M. Acciarri et al., Phys. Lett. B 411 (1997) 373.

[27] S. Catani et al., Phys. Lett. B 269 (1991) 432;

S. Bethke et al., Nucl. Phys. B 370 (1992) 310. 


\section{The L3 Collaboration:}

M.Acciarri ${ }^{26}$ P.Achard ${ }^{19}$ O.Adriani, ${ }^{16}$ M.Aguilar-Benitez ${ }^{25}$ J.Alcaraz ${ }^{25}$ G.Alemanni, ${ }^{22}$ J.Allaby, ${ }^{17}$ A.Aloisio, ${ }^{28}$ M.G.Alviggi, ${ }^{28}$ G.Ambrosi ${ }^{19}$ H.Anderhub,8 ${ }^{48}$.P.Andreev, ${ }^{6,}$ T.Angelescu, ${ }^{12}$ F.Anselmo, A.Arefiev, ${ }^{27}$ T.Azemoon, $^{3}$ T.Aziz ${ }^{10}$ P.Bagnaia, ${ }^{35}$ A.Bajo, ${ }^{25}$ L.Baksay, ${ }^{43}$ A.Balandras, ${ }^{4}$ S.V.Baldew, ${ }^{2}$ S.Banerjee ${ }^{10}$ Sw.Banerjee, ${ }^{10}$

A.Barczyk ${ }^{48,46}$ R.Barillère, ${ }^{17}$ P.Bartalini ${ }^{22}$ M.Basile, ${ }^{9}$ R.Battiston ${ }^{32}$ A.Bay ${ }^{22}$ F.Becattini ${ }^{16}$ U.Becker ${ }^{14}$ F.Behner ${ }^{48}$ L.Bellucci, ${ }^{16}$ R.Berbeco, J.Berdugo, ${ }^{25}$ P.Berges, ${ }^{14}$ B.Bertucci, ${ }^{32}$ B.L.Betev, ${ }^{48}$ S.Bhattacharya, ${ }^{10}$ M.Biasini, ${ }^{32}$ M.Biglietti, ${ }^{28}$ A.Biland, ${ }^{48}$ J.J.Blaising, ${ }^{4}$ S.C.Blyth, ${ }^{33}$ G.J.Bobbink, ${ }^{2}$ A.Böhm, ${ }^{1}$ L.Boldizsar, ${ }^{13}$ B.Borgia, ${ }^{35}$ D.Bourilkov, ${ }^{48}$ M.Bourquin, ${ }^{19}$ S.Braccini, ${ }^{19}$ J.G.Branson, ${ }^{39}$ F.Brochu, ${ }^{4}$ A.Buffini, ${ }^{16}$ A.Buijs, ${ }^{44}$ J.D.Burger ${ }^{14}$ W.J.Burger, ${ }^{32}$ X.D.Cai ${ }^{14}$ M.Capell ${ }^{14}$ G.Cara Romeo, G.Carlino, ${ }^{28}$ A.M.Cartacci ${ }^{16}{ }^{9}$ J.Casaus ${ }^{25}$ G.Castellini, ${ }^{16}$ F.Cavallari, ${ }^{35}$ N.Cavallo, ${ }^{37}$ C.Cecchi, ${ }^{32}$ M.Cerrada, ${ }^{25}$ F.Cesaroni ${ }^{23}$ M.Chamizo, ${ }^{19}$ Y.H.Chang, ${ }^{50}$ U.K.Chaturvedi, ${ }^{18}$ M.Chemarin, ${ }^{24}$ A.Chen, ${ }^{50}$ G.Chen, G.M.Chen, H.F.Chen, ${ }^{20}$ H.S.Chen? G.Chiefari ${ }^{28}$ L.Cifarelli, ${ }^{38}$ F.Cindolo, C.Civinini ${ }^{16}{ }^{\text {I.Clare}}{ }^{14}$ R.Clare ${ }^{14}$ G.Coignet, ${ }^{4}$ N.Colino, ${ }^{25}$ S.Costantini, ${ }^{5}$ F.Cotorobai, ${ }^{12}$ B.de la Cruz, ${ }^{25}$ A.Csilling, ${ }^{13}$ S.Cucciarelli, ${ }^{32}$ T.S.Dai ${ }^{14}$ J.A.van Dalen ${ }^{30}$ R.D'Alessandro ${ }^{16}$ R.de Asmundis, ${ }^{28}$ P.Déglon, ${ }^{19}$ A.Degré, ${ }^{4}$ K.Deiters, ${ }^{46}$ D.della Volpe, ${ }^{28}$ E.Delmeire, ${ }^{19}$ P.Denes, ${ }^{34}$ F.DeNotaristefani, ${ }^{35}$ A.De Salvo, ${ }^{48}$ M.Diemoz ${ }^{35}$ M.Dierckxsens, D.van Dierendonck, C.Dionisi, ${ }^{35}$ M.Dittmar, ${ }^{48}$ A.Dominguez, ${ }^{39}$ A.Doria, ${ }^{28}$ M.T.Dova, ${ }^{18, \sharp}$ D.Duchesneau, D.Dufournaud, P.Duinker, I.Duran, ${ }^{40}$ H.El Mamouni ${ }^{24}$ A.Engler ${ }^{33}$ F.J.Eppling, ${ }^{14}$ F.C.Erné, 2 P.Extermann, ${ }^{19}$ M.Fabre, ${ }^{46}$ M.A.Falagan, ${ }^{25}$ S.Falciano, ${ }^{35,17}$ A.Favara ${ }^{17}{ }^{2}$ J.Fay, ${ }^{24}$ O.Fedin ${ }^{36}{ }^{36}$ M.Felcini, ${ }^{4}$ T.Ferguson, ${ }^{33}$ H.Fesefeldt, E.Fiandrini, ${ }^{32}$ J.H.Field ${ }^{19}$ F.Filthaut ${ }^{17}$ P.H.Fisher ${ }^{14}{ }^{1}$ I.Fisk ${ }^{39}$ G.Forconi, ${ }^{14}$ K.Freudenreich,${ }^{48}$ C.Furetta, ${ }^{26}$ Yu.Galaktionov, ${ }^{27,14}$ S.N.Ganguli, ${ }^{10}$ P.Garcia-Abia, ${ }^{5}$ M.Gataullin, ${ }^{31}$ S.S.Gau, ${ }^{11}$ S.Gentile, ${ }^{35,17}$ N.Gheordanescu, ${ }^{12}$ S.Giagu, ${ }^{35}$ Z.F.Gong, ${ }^{20}$ G.Grenier, ${ }^{24}$ O.Grimm, ${ }^{48}$ M.W.Gruenewald, ${ }^{8}$ M.Guida, ${ }^{38}$ R.van Gulik, ${ }^{2}$ V.K.Gupta, ${ }^{34}$ A.Gurtu ${ }^{10}$ L.J.Gutay, ${ }^{45}$ D.Haas, ${ }^{5}$ A.Hasan, ${ }^{29}$ D.Hatzifotiadou, ${ }^{9}$ T.Hebbeker, ${ }^{8}$ A.Hervé, ${ }^{17}$ P.Hidas, ${ }^{13}$ J.Hirschfelder, ${ }^{33}$ H.Hofer, ${ }^{48}$ G. Holzner, ${ }^{48}$ H.Hoorani, ${ }^{33}$ S.R.Hou, ${ }^{50}$ Y.Hu, ${ }^{30}$ I.Iashvili, ${ }^{47}$ B.N.Jin, ${ }^{7}$ L.W.Jones, ${ }^{3}$ P.de Jong, ${ }^{2}$ I.Josa-Mutuberría, ${ }^{25}$ R.A.Khan, ${ }^{18}$ M.Kaur ${ }^{18, \diamond}$ M.N.Kienzle-Focacci, ${ }^{19}$ D.Kim, ${ }^{35}$ J.K.Kim, ${ }^{42}$ J.Kirkby, ${ }^{17}$ D.Kiss, ${ }^{13}$ W.Kittel ${ }^{30}$ A.Klimentov, ${ }^{14,27}$ A.C.König, ${ }^{30}$ A.Kopp, ${ }^{47}$ V.Koutsenko, ${ }^{14,27}$ M.Kräber $^{48}$ R.W.Kraemer, ${ }^{33}$ W.Krenz, A.Krüger ${ }^{47}$ A.Kunin, ${ }^{14,27}$ P.Ladron de Guevara, ${ }^{25}$ I.Laktineh $^{24}$ G.Landi, ${ }^{16}$ M.Lebeau, ${ }^{17}$ A.Lebedev, ${ }^{14}$ P.Lebrun ${ }^{24}$ P.Lecomte, ${ }^{48}$ P.Lecoq, ${ }^{17}$ P.Le Coultre, ${ }^{48}$ H.J.Lee, ${ }^{8}$ J.M.Le Goff, ${ }^{17}$ R.Leiste, ${ }^{47}$ P.Levtchenko, ${ }^{36}$ C.Lii, ${ }^{20}$ S.Likhoded, ${ }^{47}$ C.H.Lin ${ }^{50}$ W.T.Lin ${ }^{50}$ F.L.Linde, L.Lista, ${ }^{28}$ Z.A.Liu, W.Lohmann, ${ }^{47}$ E.Longo, ${ }^{35}$ Y.S.Lu, K.Lübelsmeyer, C.Luci ${ }^{17,35}$ D.Luckey ${ }^{14}$ L.Lugnier ${ }^{24}$ L.Luminari ${ }^{35}$ W.Lustermann ${ }^{48}$ W.G.Ma ${ }^{20}$ M.Maity ${ }^{10}$ L.Malgeri ${ }^{17}$ A.Malinin, ${ }^{17}$ C.Maña, ${ }^{25}$ D.Mangeol, ${ }^{30}$ J.Mans, ${ }^{34}$ G.Marian, ${ }^{15}$ J.P.Martin, ${ }^{24}$ F.Marzano, ${ }^{35}$ K.Mazumdar, ${ }^{10}$ R.R.McNeil ${ }^{6}$ S.Mele ${ }^{17}$ L.Merola, ${ }^{28}$ M.Meschini, ${ }^{16}$ W.J.Metzger ${ }^{30}$ M.von der Mey, A.Mihul ${ }^{12}$ H.Milcent ${ }^{17}$ G.Mirabelli, ${ }^{35}$ J.Mnich, ${ }^{17}$ G.B.Mohanty, ${ }^{10}{ }^{\text {T.Moulik, }}{ }^{10}$ G.S.Muanza, ${ }^{24}$ A.J.M.Muijs, ${ }^{2}$ B.Musicar, ${ }^{39}$ M.Musy, ${ }^{35}$ M.Napolitano, ${ }^{28}$ F.Nessi-Tedaldi, ${ }^{48}$ H.Newman, ${ }^{31}$ T.Niessen, 1 A.Nisati ${ }^{35}$ H.Nowak, ${ }^{47}$ R.Ofierzynski, ${ }^{48}$ G.Organtini ${ }^{35}$ A.Oulianov ${ }^{27}$ C.Palomares ${ }^{25}$ D.Pandoulas, ${ }^{1}$ S.Paoletti, ${ }^{35,17}$ P.Paolucci ${ }^{28}$ R.Paramatti, ${ }^{35}$ H.K.Park, ${ }^{33}$ I.H.Park ${ }^{42}$ G.Passaleva, ${ }^{17}$ S.Patricelli, ${ }^{28}$ T.Paul, ${ }^{11}$ M.Pauluzzi, ${ }^{32}$ C.Paus, ${ }^{17}$ F.Pauss ${ }^{48}$ M.Pedace ${ }^{35}$ S.Pensotti, ${ }^{26}$ D.Perret-Gallix, B.Petersen ${ }^{30}$ D.Piccolo, ${ }^{28}$ F.Pierella, M.Pieri, ${ }^{16}$ P.A.Piroué, ${ }^{34}$ E.Pistolesi, ${ }^{26}$ V.Plyaskin ${ }^{27}$ M.Pohl, ${ }^{19}$ V.Pojidaev ${ }^{27,16}$ H.Postema, ${ }^{14}$ J.Pothier, ${ }^{17}$ D.O.Prokofiev, ${ }^{45}$ D.Prokofiev ${ }^{36}$ J.Quartieri ${ }^{38}$ G.Rahal-Callot, ${ }^{48,17}$ M.A.Rahaman ${ }^{10}$ P.Raics ${ }^{15}$ N.Raja, ${ }^{10}$ R.Ramelli ${ }^{48}$ P.G.Rancoita ${ }^{26}$ R.Ranieri ${ }^{16}$ A.Raspereza ${ }^{47}$ G.Raven ${ }^{39}$ P.Razis, ${ }^{29}$ D.Ren ${ }^{48}$ M.Rescigno, ${ }^{35}$ S.Reucroft, ${ }^{11}$ S.Riemann, ${ }^{47}$ K.Riles, J.Rodin, ${ }^{43}$ B.P.Roe, L.Romero, ${ }^{25}$ A.Rosca, S.Rosier-Lees, J.A.Rubio, ${ }^{17}$ G.Ruggiero, ${ }^{16}$ H.Rykaczewski ${ }^{48}$ S.Saremi, ${ }^{6}$ S.Sarkar ${ }^{35}$ J.Salicio, ${ }^{17}$ E.Sanchez ${ }^{17}$ M.P.Sanders, ${ }^{30}$ M.E.Sarakinos, ${ }^{21}$ C.Schäfer ${ }^{17}$ V.Schegelsky ${ }^{36}$ S.Schmidt-Kaerst, ${ }^{1}$ D.Schmitz ${ }^{1}$ H.Schopper ${ }^{49}$ D.J.Schotanus ${ }^{30}$ G.Schwering, C.Sciacca, ${ }^{28}$ A.Seganti, ${ }^{9}$ L.Servoli ${ }^{32}{ }^{\text {S.Shevchenko}}{ }^{31}$ N.Shivarov, ${ }^{41}$ V.Shoutko, ${ }^{27}$ E.Shumilov ${ }^{27}$ A.Shvorob, ${ }^{31}$ T.Siedenburg, 1 D.Son, ${ }^{42}$ B.Smith, ${ }^{33}$ P.Spillantini, ${ }^{16}$ M.Steuer, ${ }^{14}$ D.P.Stickland ${ }^{34}$ A.Stone, B.Stoyanov, ${ }^{41}$ A.Straessner, K.Sudhakar, ${ }^{10}$ G.Sultanov, ${ }^{18}$ L.Z.Sun ${ }^{20}$ H.Suter, ${ }^{48}$ J.D.Swain, ${ }^{18}$ Z.Szillasi, ${ }^{43,}$ T.Sztaricskai, ${ }^{4,}$, X.W.Tang, L.Tauscher, ${ }^{5}$ L.Taylor ${ }^{11}$ B.Tellilii, ${ }^{24}$ C.Timmermans, ${ }^{30}$ Samuel C.C.Ting, ${ }^{14}$ S.M.Ting, ${ }^{14}$ S.C.Tonwar, ${ }^{10}$ J.Tóth, ${ }^{13}$ C.Tully, ${ }^{17}$ K.L.Tung, ${ }^{7}$ Y.Uchida ${ }^{14}$ J.Ulbricht, ${ }^{48}$ E.Valente ${ }^{35}$ G.Vesztergombi ${ }^{13}$ I.Vetlitsky $^{27}$ D.Vicinanza, ${ }^{38}$ G.Viertel ${ }^{48}{ }^{\text {S.Villa, }}{ }^{11}$ M.Vivargent, ${ }^{4}$ S.Vlachos, ${ }^{5}$ I.Vodopianov, ${ }^{36}$ H.Vogel $^{33}{ }^{2}$ H.Vogt,${ }^{47}$ I.Vorobiev, ${ }^{27}$ A.A.Vorobyov, ${ }^{36}$ A.Vorvolakos, ${ }^{29}$ M.Wadhwa, ${ }^{5}$ W.Wallraff, ${ }^{1}$ M.Wang, ${ }^{14}$ X.L.Wang, ${ }^{20}$ Z.M.Wang, ${ }^{20}$ A.Weber, ${ }^{1}$ M.Weber, P.Wienemann, H.Wilkens, ${ }^{30}$ S.X.Wu, ${ }^{14}$ S.Wynhoff, ${ }^{17}$ L.Xia, ${ }^{31}$ Z.Z.Xu, ${ }^{20}$ J.Yamamoto, ${ }^{3}$ B.Z.Yang, ${ }^{20}$ C.G.Yang, H.J.Yang, M.Yang, J.B.Ye, ${ }^{70}$ S.C.Yeh, ${ }^{51}$ An.Zalite, ${ }^{36}$ Yu.Zalite, ${ }^{36}$ Z.P.Zhang, ${ }^{20}$ G.Y.Zhu, R.Y.Zhu, ${ }^{31}$ A.Zichichi, ${ }^{9,17,18}$ G.Zilizi, ${ }^{43,9}$ B.Zimmermann, ${ }^{48}$ M.Zöller. 
1 I. Physikalisches Institut, RWTH, D-52056 Aachen, FRG ${ }^{\S}$

III. Physikalisches Institut, RWTH, D-52056 Aachen, FRG ${ }^{\S}$

2 National Institute for High Energy Physics, NIKHEF, and University of Amsterdam, NL-1009 DB Amsterdam, The Netherlands

3 University of Michigan, Ann Arbor, MI 48109, USA

4 Laboratoire d'Annecy-le-Vieux de Physique des Particules, LAPP,IN2P3-CNRS, BP 110, F-74941 Annecy-le-Vieux CEDEX, France

5 Institute of Physics, University of Basel, CH-4056 Basel, Switzerland

6 Louisiana State University, Baton Rouge, LA 70803, USA

7 Institute of High Energy Physics, IHEP, 100039 Beijing, China ${ }^{\triangle}$

8 Humboldt University, D-10099 Berlin, FRG ${ }^{\S}$

9 University of Bologna and INFN-Sezione di Bologna, I-40126 Bologna, Italy

10 Tata Institute of Fundamental Research, Bombay 400 005, India

11 Northeastern University, Boston, MA 02115, USA

12 Institute of Atomic Physics and University of Bucharest, R-76900 Bucharest, Romania

13 Central Research Institute for Physics of the Hungarian Academy of Sciences, H-1525 Budapest 114, Hungary ${ }^{\ddagger}$

14 Massachusetts Institute of Technology, Cambridge, MA 02139, USA

15 KLTE-ATOMKI, H-4010 Debrecen, Hungary $₫$

16 INFN Sezione di Firenze and University of Florence, I-50125 Florence, Italy

17 European Laboratory for Particle Physics, CERN, CH-1211 Geneva 23, Switzerland

18 World Laboratory, FBLJA Project, CH-1211 Geneva 23, Switzerland

19 University of Geneva, CH-1211 Geneva 4, Switzerland

20 Chinese University of Science and Technology, USTC, Hefei, Anhui 230 029, China ${ }^{\triangle}$

21 SEFT, Research Institute for High Energy Physics, P.O. Box 9, SF-00014 Helsinki, Finland

22 University of Lausanne, CH-1015 Lausanne, Switzerland

23 INFN-Sezione di Lecce and Universitá Degli Studi di Lecce, I-73100 Lecce, Italy

24 Institut de Physique Nucléaire de Lyon, IN2P3-CNRS,Université Claude Bernard, F-69622 Villeurbanne, France

25 Centro de Investigaciones Energéticas, Medioambientales y Tecnologícas, CIEMAT, E-28040 Madrid, Spainb

26 INFN-Sezione di Milano, I-20133 Milan, Italy

27 Institute of Theoretical and Experimental Physics, ITEP, Moscow, Russia

28 INFN-Sezione di Napoli and University of Naples, I-80125 Naples, Italy

29 Department of Natural Sciences, University of Cyprus, Nicosia, Cyprus

30 University of Nijmegen and NIKHEF, NL-6525 ED Nijmegen, The Netherlands

31 California Institute of Technology, Pasadena, CA 91125, USA

32 INFN-Sezione di Perugia and Universitá Degli Studi di Perugia, I-06100 Perugia, Italy

33 Carnegie Mellon University, Pittsburgh, PA 15213, USA

34 Princeton University, Princeton, NJ 08544, USA

35 INFN-Sezione di Roma and University of Rome, "La Sapienza", I-00185 Rome, Italy

36 Nuclear Physics Institute, St. Petersburg, Russia

37 INFN-Sezione di Napoli and University of Potenza, I-85100 Potenza, Italy

38 University and INFN, Salerno, I-84100 Salerno, Italy

39 University of California, San Diego, CA 92093, USA

40 Dept. de Fisica de Particulas Elementales, Univ. de Santiago, E-15706 Santiago de Compostela, Spain

41 Bulgarian Academy of Sciences, Central Lab. of Mechatronics and Instrumentation, BU-1113 Sofia, Bulgaria

42 Laboratory of High Energy Physics, Kyungpook National University, 702-701 Taegu, Republic of Korea

43 University of Alabama, Tuscaloosa, AL 35486, USA

44 Utrecht University and NIKHEF, NL-3584 CB Utrecht, The Netherlands

45 Purdue University, West Lafayette, IN 47907, USA

46 Paul Scherrer Institut, PSI, CH-5232 Villigen, Switzerland

47 DESY, D-15738 Zeuthen, FRG

48 Eidgenössische Technische Hochschule, ETH Zürich, CH-8093 Zürich, Switzerland

49 University of Hamburg, D-22761 Hamburg, FRG

50 National Central University, Chung-Li, Taiwan, China

51 Department of Physics, National Tsing Hua University, Taiwan, China

$\S$ Supported by the German Bundesministerium für Bildung, Wissenschaft, Forschung und Technologie

$\ddagger$ Supported by the Hungarian OTKA fund under contract numbers T019181, F023259 and T024011.

I Also supported by the Hungarian OTKA fund under contract numbers T22238 and T026178.

b Supported also by the Comisión Interministerial de Ciencia y Tecnología.

\# Also supported by CONICET and Universidad Nacional de La Plata, CC 67, 1900 La Plata, Argentina.

$\diamond$ Also supported by Panjab University, Chandigarh-160014, India.

$\triangle$ Supported by the National Natural Science Foundation of China. 

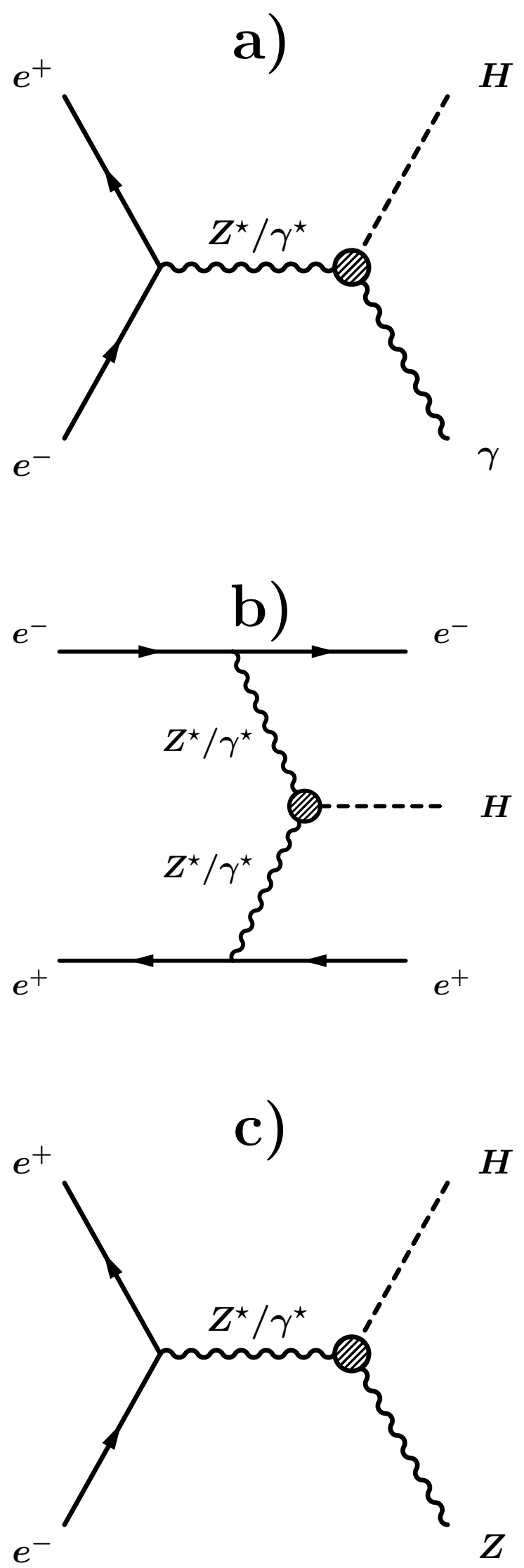

Figure 1: Relevant processes in the search for $\mathrm{H} \gamma \gamma, \mathrm{HZ} \gamma$ and $\mathrm{HZZ}$ anomalous couplings at LEP: a) $\left.\mathrm{e}^{+} \mathrm{e}^{-} \rightarrow \mathrm{H} \gamma, \mathrm{b}\right) \mathrm{e}^{+} \mathrm{e}^{-} \rightarrow \mathrm{He}^{+} \mathrm{e}^{-}$and c) $\mathrm{e}^{+} \mathrm{e}^{-} \rightarrow \mathrm{HZ}$. 

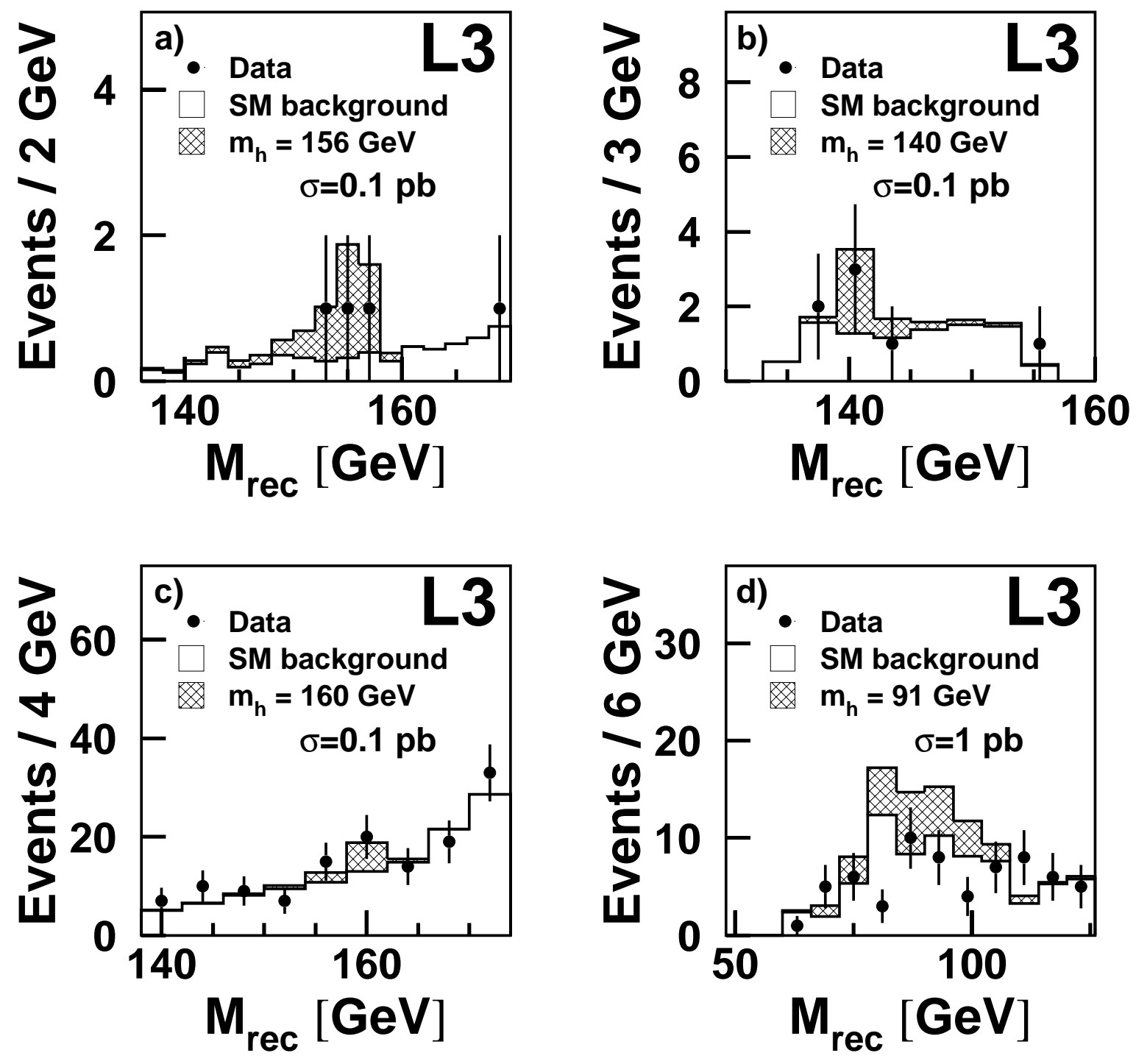

Figure 2: Distributions of the reconstructed Higgs mass, $\mathrm{M}_{\mathrm{rec}}$, for a) $\mathrm{e}^{+} \mathrm{e}^{-} \rightarrow \mathrm{H} \gamma \rightarrow \gamma \gamma \gamma$, b) $\left.\mathrm{e}^{+} \mathrm{e}^{-} \rightarrow \mathrm{H} \gamma \rightarrow \mathrm{b} \bar{b} \gamma, \mathrm{c}\right) \mathrm{e}^{+} \mathrm{e}^{-} \rightarrow \mathrm{e}^{+} \mathrm{e}^{-} \mathrm{H} \rightarrow \mathrm{e}^{+} \mathrm{e}^{-} \gamma \gamma$ and $\left.\mathrm{d}\right) \mathrm{e}^{+} \mathrm{e}^{-} \rightarrow \mathrm{e}^{+} \mathrm{e}^{-} \mathrm{H} \rightarrow \mathrm{e}^{+} \mathrm{e}^{-} \mathrm{b} \overline{\mathrm{b}}$ event candidates. The data are compared with the $\mathrm{MC}$ expectations in the presence of an anomalous Higgs signal. a), b) and c) correspond to Higgs mass hypotheses with a relevant signal significance. d) illustrates the large suppression of $\mathrm{e}^{+} \mathrm{e}^{-} \rightarrow \mathrm{Z} \gamma$ background in the $\mathrm{e}^{+} \mathrm{e}^{-} \rightarrow$ $\mathrm{e}^{+} \mathrm{e}^{-} \mathrm{H} \rightarrow \mathrm{e}^{+} \mathrm{e}^{-} \mathrm{b} \overline{\mathrm{b}}$ selected sample, as no large peak structure is observed at the $\mathrm{Z}$ mass. 


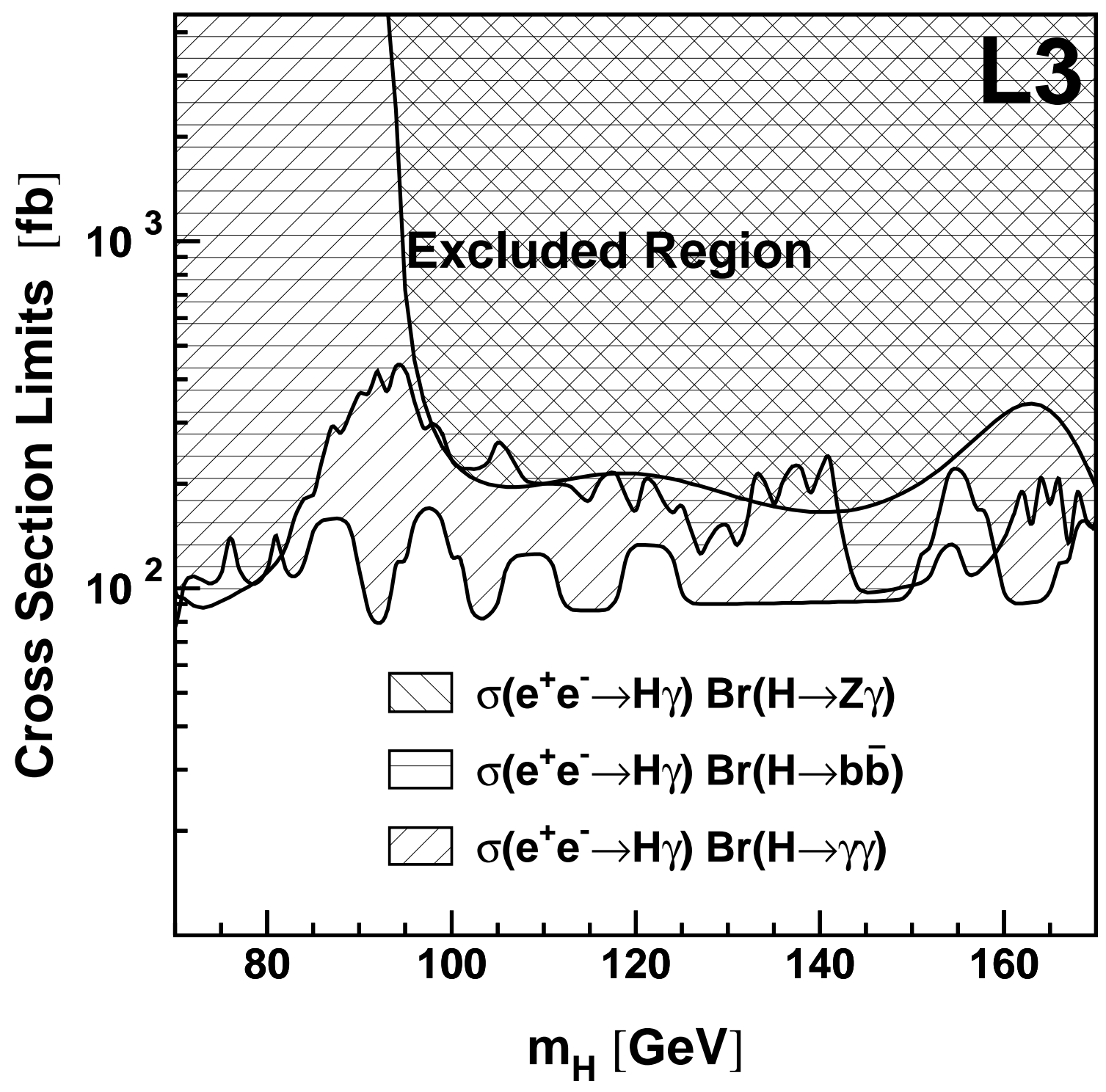

Figure 3: Cross section 95\% CL upper limits as a function of the Higgs mass, $m_{\mathrm{H}}$, for the $\mathrm{e}^{+} \mathrm{e}^{-} \rightarrow \mathrm{H} \gamma \rightarrow \gamma \gamma \gamma, \mathrm{e}^{+} \mathrm{e}^{-} \rightarrow \mathrm{H} \gamma \rightarrow \mathrm{b} \overline{\mathrm{b}} \gamma$ and $\mathrm{e}^{+} \mathrm{e}^{-} \rightarrow \mathrm{H} \gamma \rightarrow \mathrm{Z} \gamma \gamma$ processes in the presence of anomalous Higgs couplings at $\sqrt{s}=189 \mathrm{GeV}$. 


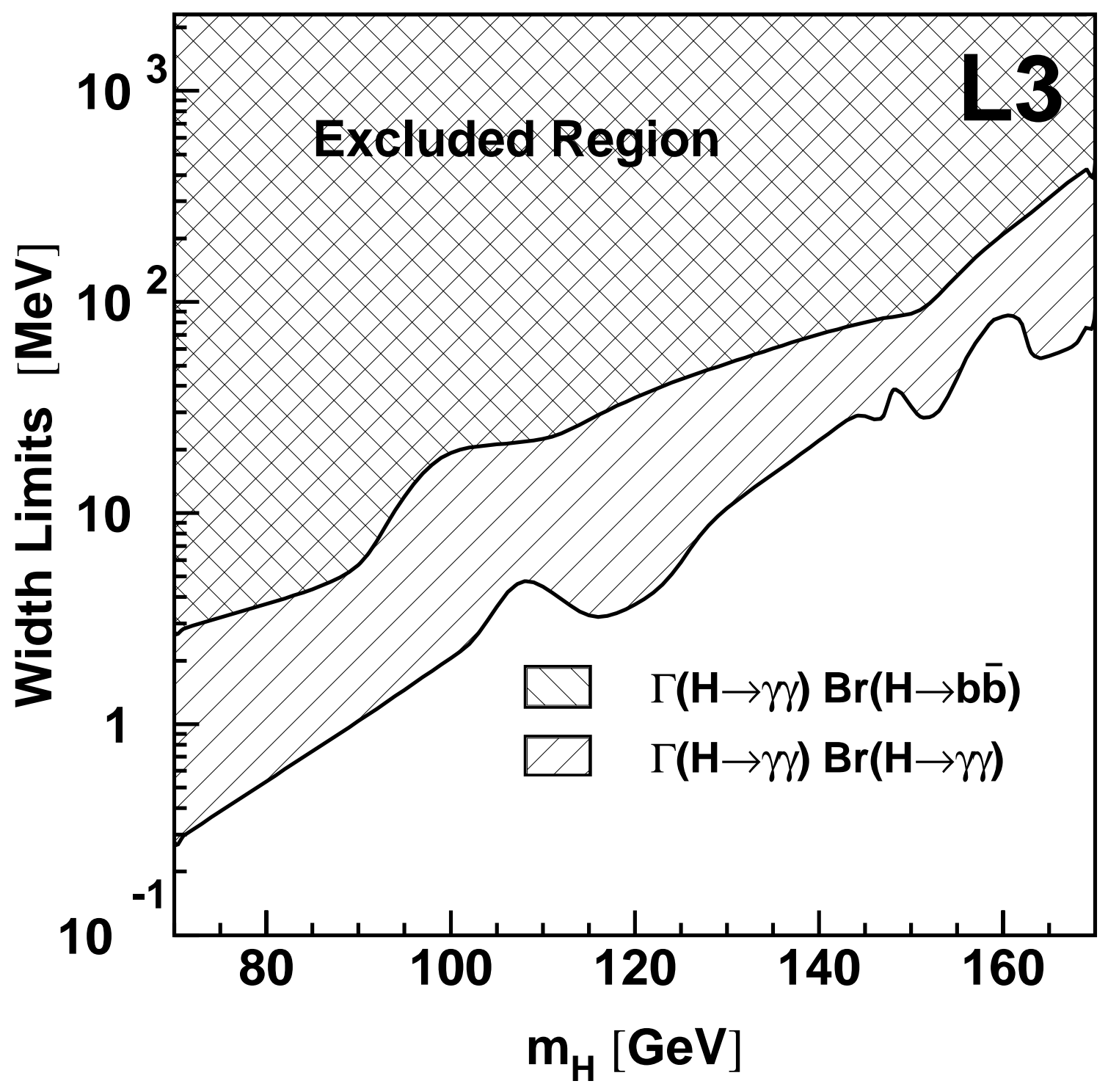

Figure 4: Upper limits at the $95 \% \mathrm{CL}$ on the quantities $\Gamma(\mathrm{H} \rightarrow \gamma \gamma) \mathrm{Br}(\mathrm{H} \rightarrow \gamma \gamma)$ and $\Gamma(\mathrm{H} \rightarrow$ $\gamma \gamma) \operatorname{Br}(\mathrm{H} \rightarrow \mathrm{b} \bar{b})$ as a function of the Higgs mass, $m_{\mathrm{H}}$, from the analysis of the $\mathrm{e}^{+} \mathrm{e}^{-} \rightarrow \mathrm{He}^{+} \mathrm{e}^{-}$ process in the presence of anomalous Higgs couplings at $\sqrt{s}=189 \mathrm{GeV}$. 

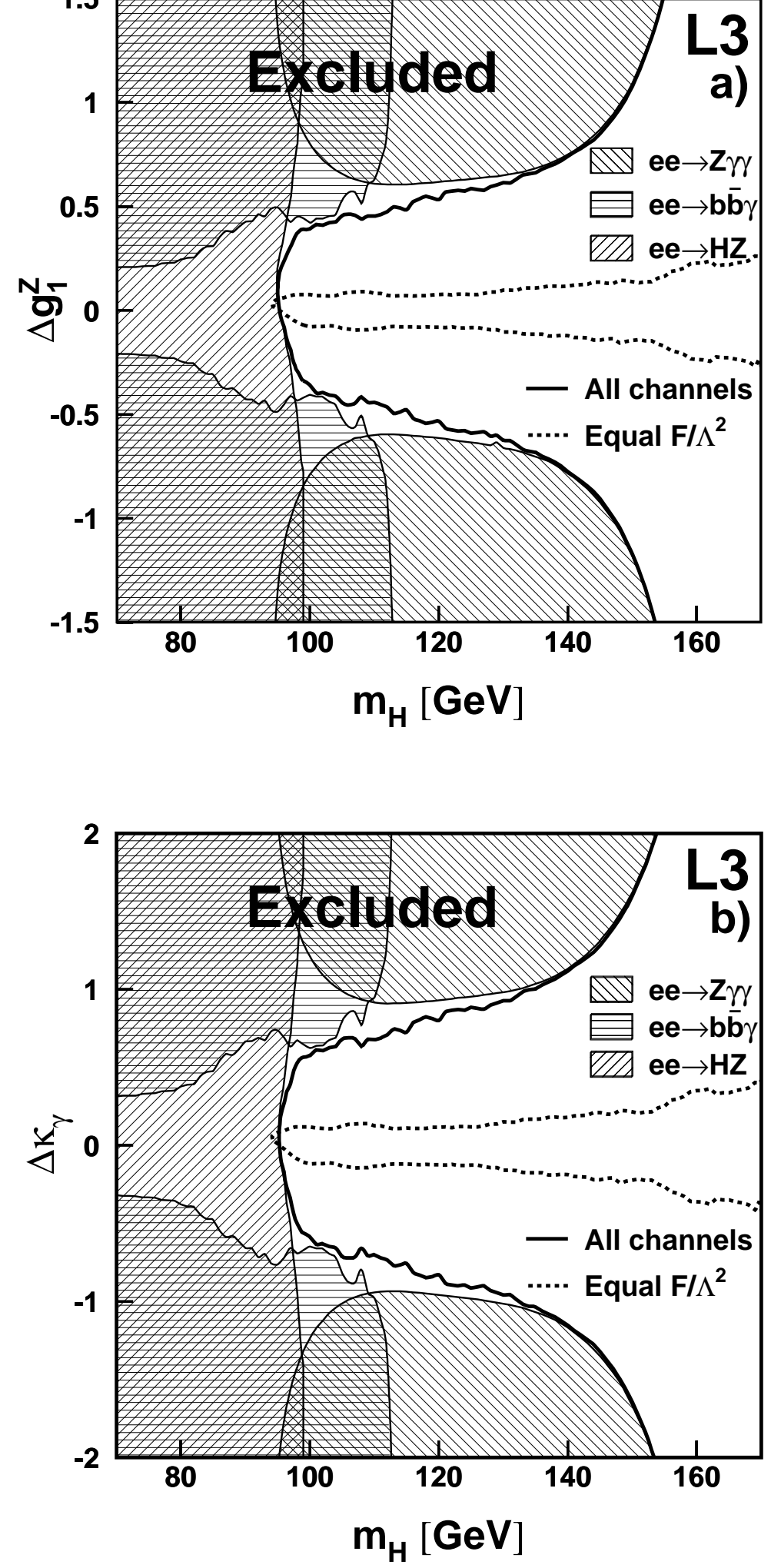

Figure 6: Excluded regions for the anomalous couplings a) $\Delta g_{1}^{\mathrm{Z}}$ and b) $\Delta \kappa_{\gamma}$ as a function of the Higgs mass $m_{\mathrm{H}}$. Limits on $\Delta g_{1}^{\mathrm{Z}}$ are obtained under the assumption $d=d_{B}=\Delta \kappa_{\gamma}=0$, while limits on $\Delta \kappa_{\gamma}$ assume the relation $d=d_{B}=\Delta g_{1}^{\mathrm{Z}}=0$. The regions excluded by the most sensitive analyses: $\mathrm{e}^{+} \mathrm{e}^{-} \rightarrow \mathrm{H} \gamma \rightarrow \mathrm{Z} \gamma \gamma, \mathrm{e}^{+} \mathrm{e}^{-} \rightarrow \mathrm{H} \gamma \rightarrow \mathrm{b} \overline{\mathrm{b}} \gamma$ and $\mathrm{e}^{+} \mathrm{e}^{-} \rightarrow \mathrm{HZ}$ are also shown. In addition, we show the limits reached under the assumption of equal couplings at the scale of new physics $\Lambda$ (dashed lines) as described in the text. 

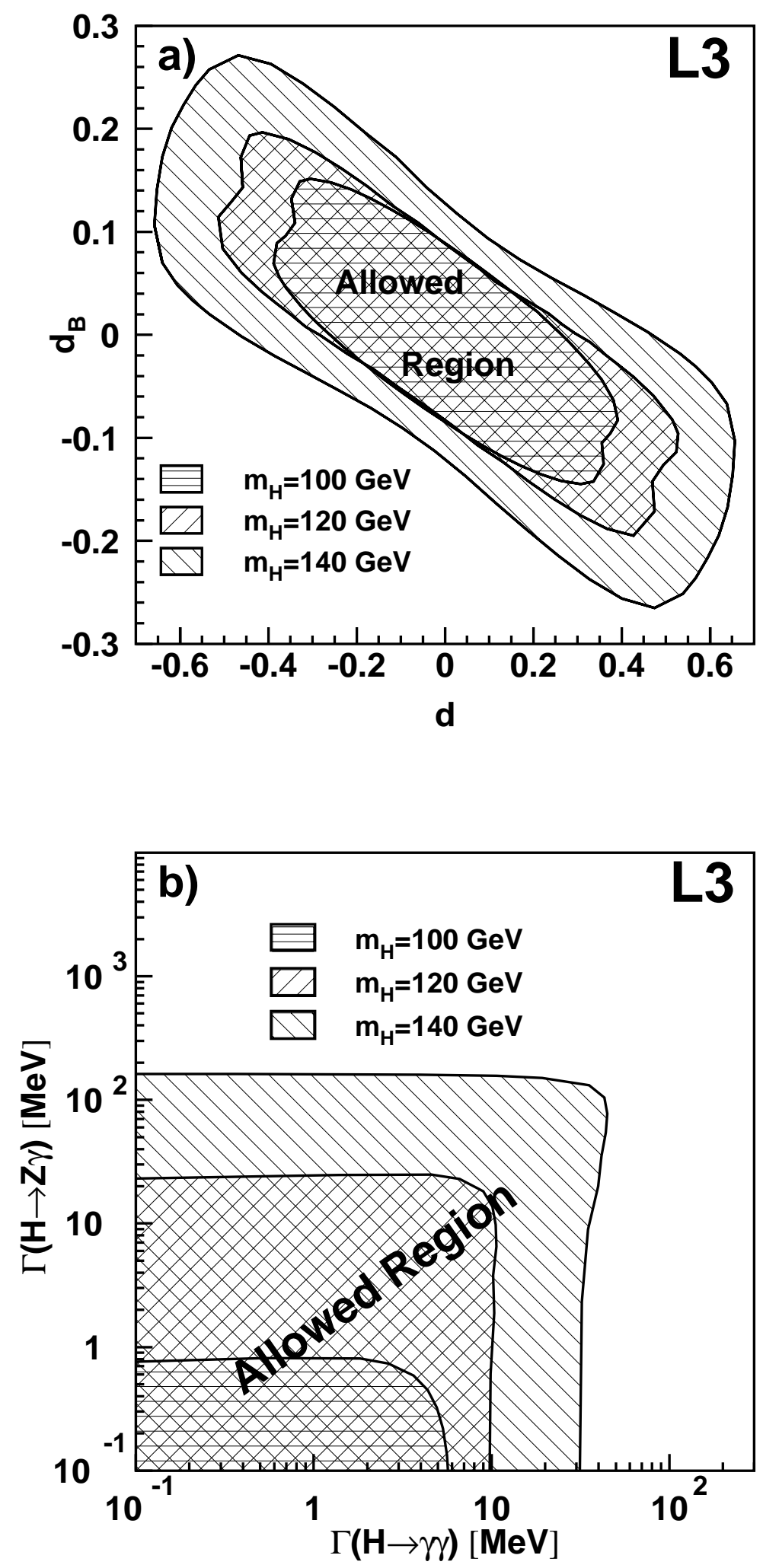

Figure 7: Allowed regions at more than $95 \% \mathrm{CL}$ in the a) $\left(d, d_{B}\right)$ and b) $(\Gamma(\mathrm{H} \rightarrow \gamma \gamma), \Gamma(\mathrm{H} \rightarrow$ $\mathrm{Z} \gamma)$ ) planes for different Higgs mass assumptions. All analyzed channels are used. The results are consistent with the SM expectations: $d \approx d_{B} \approx 0$ and $\Gamma(\mathrm{H} \rightarrow \gamma \gamma) \approx \Gamma(\mathrm{H} \rightarrow \mathrm{Z} \gamma) \approx 0$. 